\title{
Hardware Simulator for Seizure, Preseizure and Normal Mode Signal Generation in LabVIEW Environment for Research
}

\author{
Uvais Qidwai, Aamir Saeed Malik, Mohamed Shakir, and Nidal Kamel
}

\begin{abstract}
Currently numerous theories and model have been developed to associate various findings or in relating EEG patterns to develop a software simulator. Here we develop a hardware simulator of the EEG model or to simulator any EEG data set in either .edf or .tdmsot .txtformat from any patient or database depository. The proposed hardware simulator will enhance researchers and hardware validators to simulate, validate and test their detection algorithms forehand, before actual testing the algorithm in the actual standalone hardware. This system make use of signal generator block and then pass this data to the external hardware data acquisition system like the NI-DAQ with an external option to transfer the data wirelessly(Bluetooth, Zigbee, Wi-Fi) or wired (analog port, serial bus etc). This simulator can simulate or generate seizure, pre-seizure and normal EEG waveform. The paired cost effective Arduino microcontroller (in case of wireless system) will be having the algorithm in built in order to classify the type of signal received. This can help in developing wearable EEG Seizure monitoring system(WBAN-HL7). Thispaper will enhance the purpose of developing a system which can alert locally in a form of wearable gadget, whenever a pre-seizure occurs. This can help the epileptic patient or the user to take precautionary action to save themselves from accidents or injury, just before the occurrence of the seizure. Useable of this embedded wearable version can ensure a better everyday activities and the psychological stress can be reduces to leverage the social interaction.
\end{abstract}

Index Terms-Bio-signal, simulator, embedded system, Bluetooth, EEG, seizure, pre-seizure.

\section{INTRODUCTION}

The EEG bio-signal hardware simulator is intended for designing, to develop algorithms, signal processing as a portable system [1], [2], by utilizing the power of LabVIEW and to implement it into an embedded Data Acquisition System (DAQ). The user can view the signal and transmit the EEG data to a remote microcontroller system for inhouse data processing and identification, this can be used for validating wearable bio signal system before implementing the actual wearable epilepsy detection system, moreover this simulator can be used as a virtual environment simulator [3]for generating EEG signals and can be used for fine tuning the and rehearse theproduct (wearable system).This can also be built on the approach of dataflow in graphical form, with the integration of hardware signal output. Thisas a platformenables us to focus on the system design, even if

Manuscript received May 10, 2013; revised July 12, 2013.

Uvais Qidwai is with the Computer Engineering Department, Qatar University, Qatar.

Aamir Saeed Malik, Mohamed Shaki, and Nidal Kamel are with the Neuro-Signal Processing Group, Centre for Intelligent Signal and Imaging Research, Universiti Teknologi PETRONAS, Tronoh, Perak, Malaysia. lots of other languages for programming are accessible for constructing or generating the analog EEG signals and the specific part of the signal. The control of a rapid transition from a one psycho-physicalstate to another [4] is easily attainable with an EEG simulator, In LabVIEW, basically the biomedical tool kit provides applications which are Ready-to-run. They in cooperate File Format Converter Player, Biosignal Datalogger, Biosignal Generator, File Viewer, Blood Pressure (BP) Analyzer, Image Reconstruction of image in 3D, Feature Extractor for ECG, Variability Analyzer for the heartbeat rate. All the common file types' conversions are possible like the TDMS extension (National Instruments Technical Data Management Streaming), including ACQ, iWorx, Biopac, .edf, .mat, .txt and HL7. This tool kit is also capable of implementing algorithms for the signal processing on EEG and ECG bio signals. It also enables signal simulation of the signal, analysis of coherence and bispectral EEG, extraction of specific feature of the ECG, and power analysis of the EMG waveform, predefined virtual simulators for electroencephalography (EEG) and electrocardiogram (ECG). This system helps for the system which can be implemented and for the investigations of tiny, one-channel electroencephalogram systems in an ambulatory setting [5]. We will be using a part of this tool kit only just to validate the LabVIEW program build specifically to test the program data flow and its functionality to generate the Seizure signal via a DAQ and to transmit to a in range microcontroller. The microcontroller reads the data from either the serial SPP port or via the inline analog to digital convertor port.

\section{METHOD AND DESIGN}

When it comes to designing algorithms for low power platforms, which is constrained by memory and computational limitations [6], simulators play an important role in optimization.First, a dedicated systemforthisintension isbuilttoimprove with respect to the current system limitations, associated to approach of hefty processing, realtime healthcare facility accessibility andproperanalysis.In the simulator, the peak positions are not invariable, but may change from one region to another [7]. This paper presents the procedure to simulate the EEG signal in LabVIEW environment and to detect the presence of healthy EEGvs. Pre-seizure/Seizure signal, here then to be detected by the cost effective microcontroller. Since there is no much realtime data on EEG patients, the selection of EEG zones are simulated with respect to the alarm priority and threestatesare alsoselectedfor the testing and execution in 
the initial prototype of the whole system. Here the program is used to simulate the abnormal EEG morphology [8], this program in the LabVIEWhas two mode of operation: 1) read the existing files recordings from a database or from the patient offline with contains normal, pre-seizure and seizure waveforms and 2) simulate a simple EEG model, when can simulate normal, pre-seizure and seizure waveform.

In Fig. 1, the system demonstrates a general setup for the experimentation and functioning flow. Here, the EEG signal waveform is fed from the LabVIEW to a NI-DAQ, which is available from commercially or for freely available data base which contains normal, pre-seizure and seizure datasets Any abnormal data reflects that there are abnormal activities in the EEG signal [9] and the program should be set to activate an alarm with respect to a set point in the program.

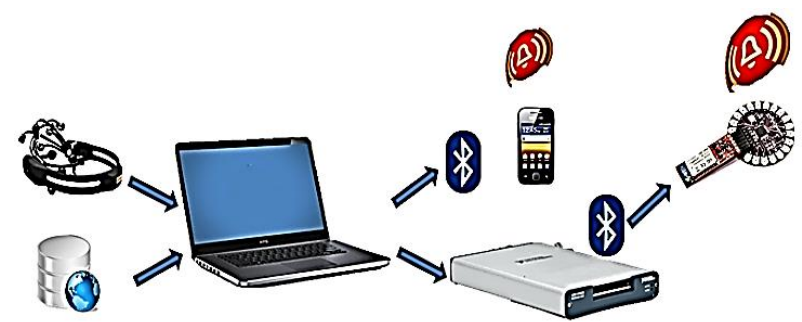

Fig. 2. Overall functional system.

\section{EXPERIMENTAL SETUP}

Byusing the LabVIEW Data Flow program, the spike wave and the multi-spike wave of the EEG morphology are reconstructed [10] from the text file. In NI X Series from National Instrumentsis aUSB compatible device for acquisition of data. This multifunctional device have high performance digital I/O, control channels, counter/timers and analog measurement onto the portable wireless microcontroller (ArduinoLilypad in this test bench case). By using this device, we can extend the applications from multiple microcontroller data-logging applications, measurement and portable test. This device has four analog outputs, thirty two analog inputs, four counters and forty eight I/O digital lines.It also has Analog input which is multiplexed simultaneous sampling up to 2 Mega Samples per second per channel. This device supported with flowchart type graphical programming with LabVIEW. In case of real time EEG acquisition of data, we can directly wire the channel signal to the analog input for post processing including classification and identification of seizure types. This device also supports more accurate triggering, advanced timing, synchronization and high speed bidirectional real time streaming of signal and data by using NI-STC3 protocol technology. This protocol supports dedicated digital Input-Output and analog subsystems with independent timing engines for parallel digital $\mathrm{I} / \mathrm{O}$ and analog to execution at different frequency synchronization with the help of 32-bit counters. This counter can also be used for encoders pulse width modulation(PWM). This device can also be used for recording and analyzing transient time.

The only disadvantage with this DAQ is that if we want to record and analyze more than 4 channels of EEG signals of the real time patients, then we have to use Analog-Digital MUX. So an additional 16 channels can be added. Digital to
Analog convertor. If we wanted to transmit more than four channels by reading from the EEG database to the microcontroller via NI-DAQ, then we have to use parallel 8/16/32 bit Digital to Analog Convertor (DAC). This depends on the number of available digital I/O pins. In this workbench, we have $32 \mathrm{I} / \mathrm{O}$ pins; therefore we can create an additional four more ADC ports with a resolution of 8/10/12 bits per channel (in case of 8-bit parallel DAC we use AD5330 from analog devices).

In the subset of the program, the abnormal EEG dataset indicates central nervous system abnormality [11], cognitive states such as alertness and arousal [12]. This system also have the option to acquire in real time via EEG headsets like 128 channel headset or 16 channel head set like the Emotiv, and then transmit to the microcontroller for validation of the algorithm developed in order to identify by classification the state of pre-seizure in order to eliminate false prediction as much as possible. Once the algorithms have identified the pre-seizure, immediately the alarm is invoked in order to take precautionary acts to save the patient/user from more injury or accidents. By using a simulator we can optimize the electrode position in the patient when it comes to implementation of standalone microcontroller system to sense the EEG signal directly from the patient. The lesser number of ADCs input to the microcontroller, the faster the response to invoke the alarm. This also helps in simplify the computational algorithms, moreover frees temporary memory inside the microcontroller.

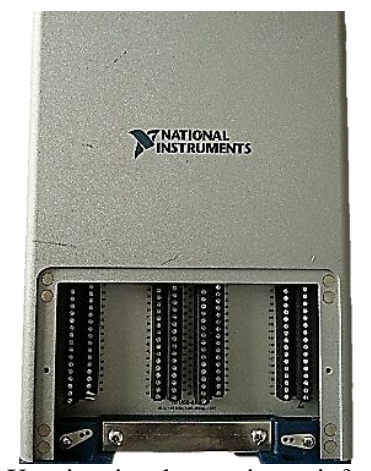

Fig. 2. NI-DAQ -X series signal genration unit for EEG simulation.

The Bluetooth module used with the DAQ and microcontroller is the Bluetooth Mate Gold modem. The data is transmitted and received by the serial (RX/TX) pipe pins. This Bluetooth device can transmit and stream data from 2400 bits per second (bps) to 115200bps to the microcontroller, with a maximum range of 300 feet distance, which will be more than sufficient for the connectivity with the DAQ or the patient carrying the microcontroller with them.Here the data from the DAQ is transferred to the microcontroller in a packetized serial data. If there is to send more than one channel data, the following packet format is followed to ensure no data have lost in the microcontroller side:

$$
\text { *adc_channel1;adc_channel2;...adc_channeln\# }
$$

The microcontroller used in this setup is the mainboard ArduinoLilypad. This PCB consists of an ATmega328, 
which can be operated from 2Volts till 5.5 Volts. This microcontroller has a surface mount crystal oscillator clocking at maximum of $8 \mathrm{MHz}$. Atmega328P from Atmel has fourteen I/0 pin in which, seven of them are ADC, six PWM pins, one serial Tx/Rx bus, $16 \mathrm{~KB}$ of flash memory, $1 \mathrm{~KB}$ of SRAM and 512 bytes of EEPROM. This type of microcontroller has a special type of design in which it can be used with e-textile wearable technology.

This module can be stitched with cloths.In the wearable end (user notification point), equipped with we have a local alarm with vibration in the microcontroller or the mobile user console to notify the patient/user the occurrence of PreSeizure state in the body of the patient.

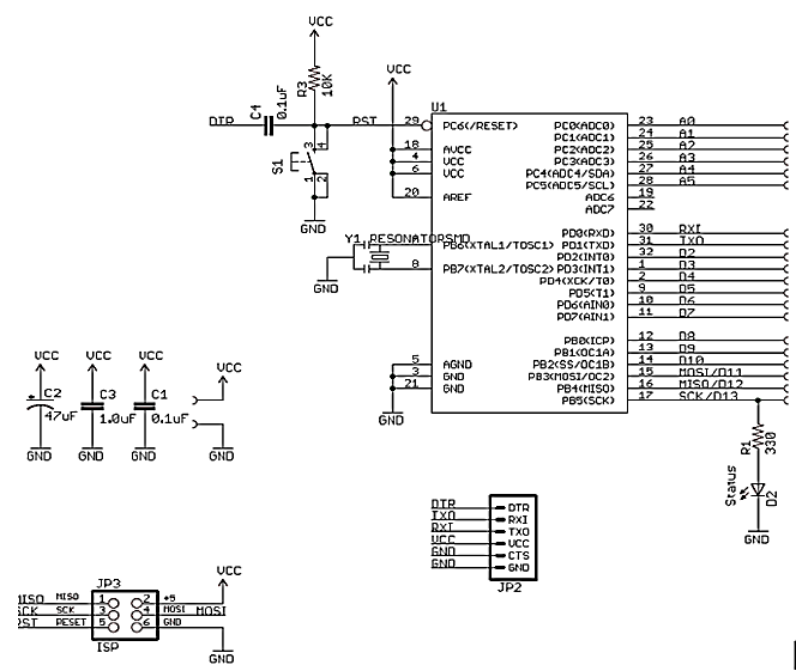

Fig. 3. Schematic of arduino lilypad mega328p used for algorithm.

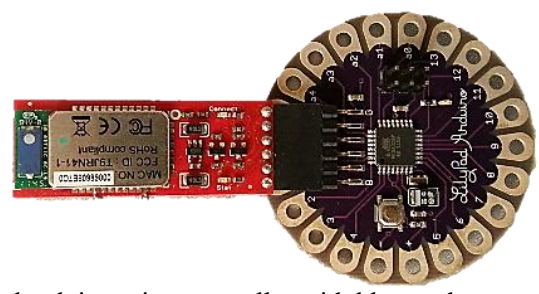

Fig. 4. Lilypad arduino microcontroller with bluetooth-mate serial modem.

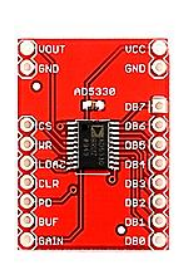

(a)

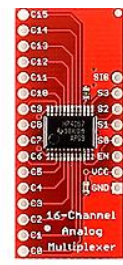

(b)
Fig. 5. Breakout board :(a)Parallel 8-Bit DAC, (b)analog/digital MUX.

In mode one (to simulate and experiment external EEG database), the EEG signal with pre-seizure and seizure patients, into the hardware DAQ, the EEG scalp data is used from CHB-MIT database. This EEG depository consists of dataset of twenty three patients (129 datasets with more than one seizure in .edf file format). The database of the EEG signal is provided by Boston children-hospital. The signals are sampledat the rate of 256 samples per second, with sixteen bits of resolution.

Since the hardware simulator emulates the patient for the microcontroller, we can specifically select the channel to be transmitted to implement the algorithm on the individual channel to find out the individual response of the channel when processed by the microcontroller. In the microcontroller part, the statistical parameters like the energy, variance, kurtosis, Skewness etc. will be calculated on individual channel depending upon the user. This enables selectivity by the microcontroller.

\section{GENERATION OF EEG SIGNAL}

The generation of signal from the EEG dataset is dependent of a number of variables [13]. This dataset changes from subject to subject and this simulator can generate any type of EEG signal. The seizure start points and end points have been clearly described in the database given by the CHB-MIT database. Before sending the test signal to the NI-DAQ hardware, the signal should be checked in the graph to select the start point of the seizure occurrence, manually by entering the values or parsing from a text file which contains an array of the file name, time (ms) and the waveform data. With respect to this, we calculate pre-seizure, seizure and normal EEG. We have arbitrary selected pre-seizure as ten seconds before the incidence of seizure, as per definedfrom the database. Twenty seconds before the seizure, isthe start point of the normal EEG.

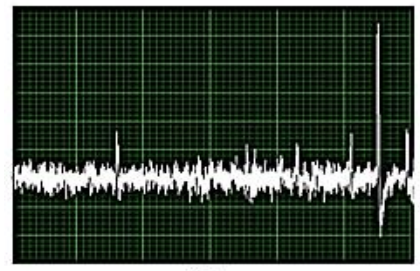

(a)

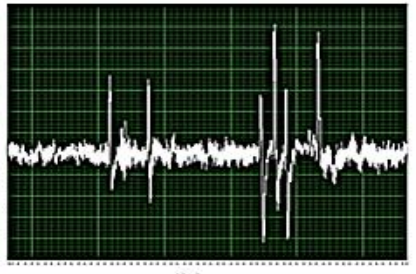

(b)

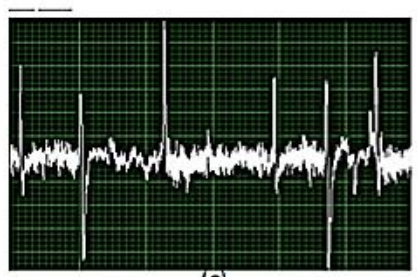

(c)

Fig. 6. Sample signal from the EEG data base in labVIEW graph: (a)nonseizure, (b) preseizure and, (c) paritial seizure.

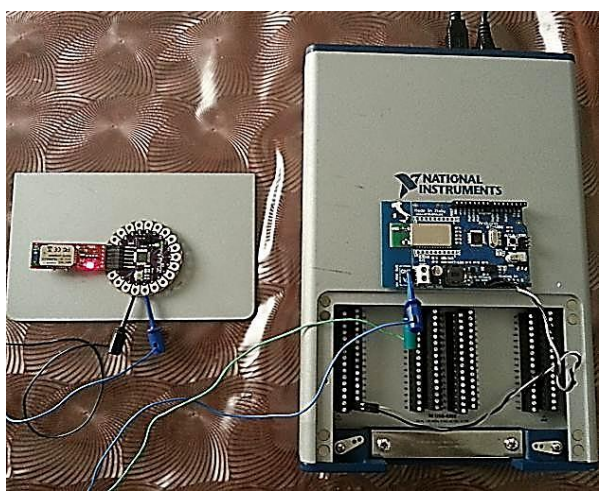

Fig. 7. EEG Simulator module; Arduino BT and Lilypad microcontroller with bluetooth receiver.

In the case of mode two : we will be simulating an artificial EEG signal raw signal with respect to a simple EEG signal model roughly by adding virtual channel mode. Here this is not imitating any of the patients EEG data There this mode can be used in cases where there is no 
access to the real EEG data for simulation. In this mode the disadvantage is that it is repetitive after each cycle is executed.

In Fig. 7, the ADC of the ArduinoBT microcontroller connected to the National Instruments DAQ ADC, which helps in transferrin the EEG waveform to the Lilypad microcontroller Bluetooth. The advantage is that it provides flexibility to the user to design our with EEG signal by using the Signal Simulator from the signal processing toolbox or toolkit. We can add noise; add standard signals (sine, sawtooth, triangular etc.) filter before sending it to the external DAQ.

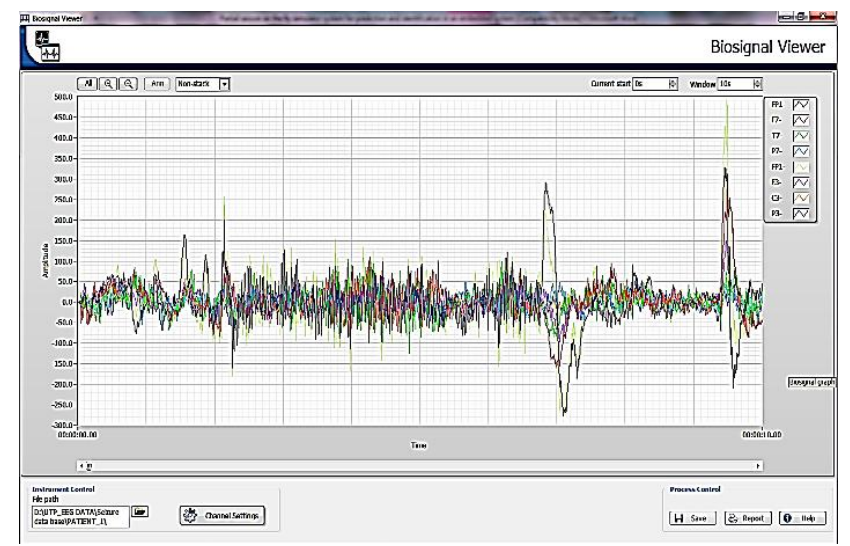

Fig. 8. Bio signal viewer : front panel-shown all the channel waveform together (normal case).

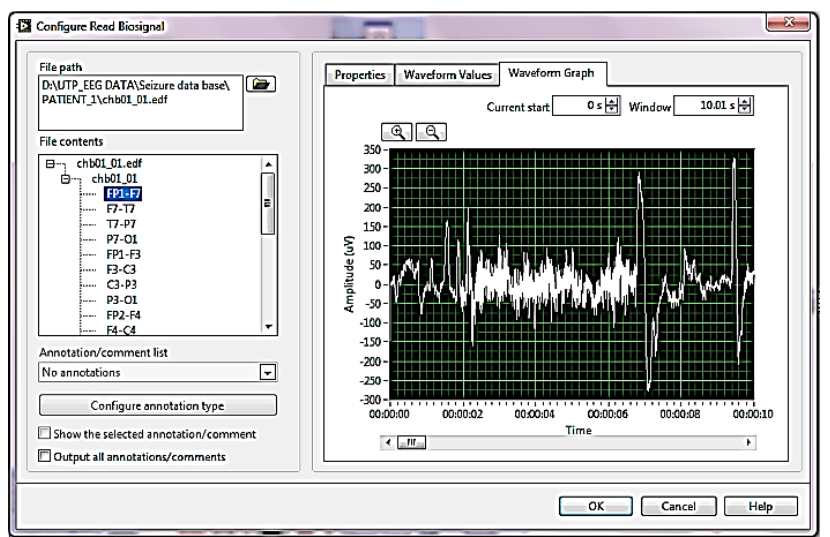

Fig. 9. Bio Signal read configuration settings panel: here only the first channel is selected with a graph window of 10.01 seconds.

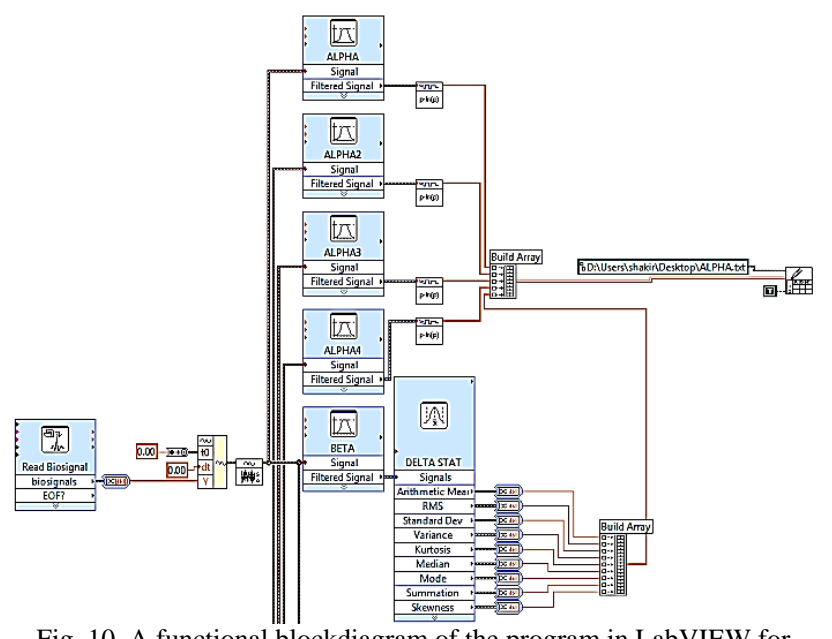

Fig. 10. A functional blockdiagram of the program in LabVIEW for filtering and saving.

We can change the frequency, and design filter if needed before transmitting to the microcontroller for classification. In this mode we can also create customized signal equations with respect the approximation model of EEG signal and generate the signal in the LabVIEW user interface program. Here in the Fig. 7, to Read the EEG signal from the database then normalize pass it to Alpha and Beta filter and calculate the statistical variables of the particular waveform and save it in .txt file for reference. Once the algorithm is finalized in the microcontroller by using the signals from the EEG simulator workbench, it can replaced with the real patient EEG data probe in real time on the fly. In this mode of operation, the arduino microcontroller will pre-process the input EEG signal from the human subject and look for a presizure state. After analysis and classification, if a preseizure state occurs, the microcontroller will alert locally and with the Bluetooth link to the mobile device. In future, we can add additional connectivity to the hospital database to transfer the time stamp of the occurrence of seizure/preseizure, location of occurrence and the EEG signal waveform itself at that time. This will help the medical doctor to look into more details about the EEG characteristics of the signal and the subject itself for further analysis by heuristics data. This software and the hardware provide support for the education and research of electrophysiology [14].

\section{CONCLUSION}

In this paper, a strategy is presented in order to modify an existing Data Acquisition/Generation module to function as an EEG simulator. Such a simulator will be very helpful in EEG related research since all the initial algorithms can be tuned to the controlled data first before going to the actual human subjects. Unlike the commercial ECG simulators, to the best of our knowledge, there is no such commercially available system that can be used for such research tasks. The use of NI device and LabVIEW environment makes it very user friendly for anyone to develop a device tailored to their requirements. The data we have used is from actual patients and the system converts the text files for the data values into electrical voltages similar to what would be measured by the EEG probes. Further work is being done in producing more simulated data based on empirical models of the real waveforms. With controlled data types, Healthy/Normal, Seizure, and Pre-Seizure classes, the user can select the type and work on tuning their algorithms for detection and classification applications.

\section{REFERENCES}

[1] X. Fan, L. Bi, and Z. Wang, "Detecting emergency situations by monitoring drivers' states from EEG," Complex Medical Engineering (CME), 2012 ICME International Conference, pp. 245-248, 1-4 July 2012.

[2] Z. Khaliliardali, R. Chavarriaga, L. A. Gheorghe, and J. R. del Millan, "Detection of anticipatory brain potentials during car driving," Engineering in Medicine and Biology Society (EMBC), 2012 Annual International Conference of the IEEE, pp. 3829-3832, Aug. 28, 2012Sept. 1, 2012.

[3] N. Katayama, K. Hidaka, A. Karashima, and M. Nakao, "Development of an immersive virtual reality system for mice," SICE Annual Conference (SICE), pp. 791-794, 20-23 Aug. 2012.

[4] A. Borgul, A. Margun, K. Zimenko, A. Kremlev, and A. Krasnov, "Intuitive control for robotic rehabilitation devices by human-machine interface with EMG and EEG signals," Methods and Models in Automation and Robotics (MMAR), pp. 308-311, 27-30 Aug. 2012. 
[5] J. Duun-Henriksen, R. E. Madsen, L. S. Remvig, C. E. Thomsen, H. B. D. Sorensen, and T. W. Kjaer, "Automatic Detection of Childhood Absence Epilepsy Seizures: Toward a Monitoring Device," Pediatric Neurology, vol. 46, issue 5, pp. 287-292, May 2012.

[6] S. Ravindran and R. Cole, "Heart rate and epileptic seizure detection algorithms for low-power platforms,"Biomedical Signal Processing and Control, vol. 7, issue 3, May 2012.

[7] Z. Ma, W. Zhou, Q. Yuan, and S. Geng, "Simulation of ictal EEG with a neuronal population model," Bioelectronics and Bioinformatics (ISBB), pp. 103-106, 3-5 Nov. 2011.

[8] W. Zhe, M. Hui, Ge. Manling, and G. Dong, "A Simulation of the Abnormal EEG Morphology by the 3-D Finite Element Method," Engineering in Medicine and Biology Society, IEEE-EMBS, pp. 36203623, 17-18 Jan. 2006.

[9] H. Meng, Z. Wang, M. Ge, X. Jiang, T. Zhang, J. Guo, and G. Dong, "An Understanding for the Abnormal Spikes of the EEG Simulation in a 2-D Neural Network," Engineering in Medicine and Biology Society, IEEE-EMBS, pp. 3008-3011, Jan. 2006.

[10] M. Ge, G. Dong, H. Meng, L. Wang, S. Yang, B. Wang, and C. Zhao, "An Abnormal EEG Simulation Based on The Chay Model of An Excitable Neuron," Neural Engineering, Conference, pp. 45-47, 1619 March 2005

[11] L. Rankine, H. Hassanpour, M. Mesbah, and B. Boashash, "Newborn EEG simulation from nonlinear analysis," in Proc. The Eighth International Symposium Signal Processing and Its Applications, pp. 191-194, August 28-31, 2005.

[12] R. C. Wu, C. T. Lin, S. F. Liang, T. Y. Huang, Y. C. Chen, and T. P. Jung, "Estimating driving performance based on EEG spectrum and fuzzy neural network," in Proc. Neural Networks, 29 July 2004.

[13] C. Janeczko and H. S. Lopes, "A genetic approach to ARMA filter synthesis for EEG signal simulation," Evolutionary Computation, pp. 373-378, vol. 1, 2000.

[14] M. J. Quist, F. Zanow, and P. J. M. Cluitmans, "Design of physiological source analysis software for educational purposes," in Proc. The 19th Annual International Conference of the IEEE Engineering in Medicine and Biology Society, vol. 3, pp. 1044-1047, vol. 3, 30 Oct. 1997.

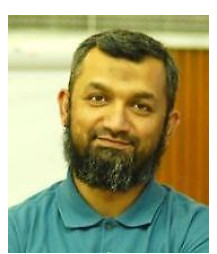

Uvais Qidwai received his $\mathrm{Ph} . \mathrm{D}(\mathrm{EE})$. from the University of Massachusetts-Dartmouth USA in 2001, MS(EE) in 1997 from KFUPM Saudi Arabia, and BS(EE) in 1994 from NED University of Engineering \& Technology, Karachi, Pakistan. He taught in the Electrical Engineering and Computer Science Department, Tulane University, in New Orleans as Assistant Professor, and was in-charge of the Robotics lab as well as a research member of Missile Defence Centre, during June 2001 to June 2005. He joined the Computer Science and Engineering Department, Qatar University, in FALL of 2005 where he is currently working as Associate Professor. Dr. Qidwai's present research interests include Robotics, Signal and Image Processing, Expert Systems design for Industrial Applications, and Intelligent Algorithms for medical informatics. He has participated in several government- and industryfunded projects in the United States, Saudi Arabia, Qatar, UAE, Singapore,
Malaysia, and Pakistan, and has published over 95 papers in reputable journals and conference proceedings.

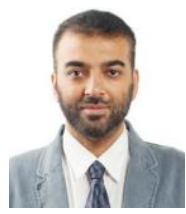

Aamir Saeed Malik has a BS in electrical engineering from University of Engineering \& Technology, Lahore, Pakistan, MS in Nuclear Engineering from Quaid-iAzam University, Islamabad, Pakistan, another MS in Information \& Communication and $\mathrm{PhD}$ in Information \& Mechatronics from Gwangju Institute of Science \& Technology, Gwangju, Republic of Korea. He has more than 10 years of research experience and has worked for GoP, IBM, and Hamdard University in Pakistan, and Yeungnam and Hanyang Universities in South Korea. He is currently working at Universiti Teknologi Petronas as Associate Professor in Malaysia. He holds the responsibility as Director at UTP-MOR (Mission Oriented Research) - Biomedical Technology. He is Senior Member of IEEE. His research interests include image processing, 3D shape recovery, medical imaging, Health informatics, EEG signal processing and content based image retrieval (CBIR).

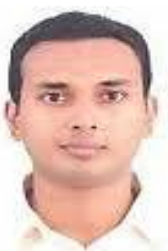

Mohamed Shakir received the B.Tech degree in Applied Electronics and Instrumentation from MES College of Engineering, Calicut University, India, in 2005 and the M.S. degree in Electrical Engineering from Washington International University, USA in 2011. He is currently pursuing the Ph.D. degree in Electrical Engineering at Univeristi Teknologi Petronas, Malaysia. From 2011 to 2014, he was a Research Assistant with the Computer Engineering Department, Qatar University, Qatar. His research interest includes Robotics, Assistive Technology, Interfacing, Bio-Signal processing of biological/medical systems.

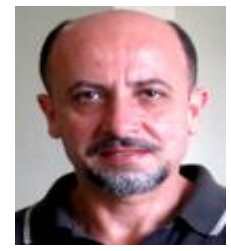

Nidal Kamel received the $\mathrm{PhD}$ degree (Hons) from the Technical University of Gdansk, Poland, in 1993. His $\mathrm{PhD}$ work was focused on subspace-based array signal processing for direction of-arrival estimation. Since 1993 he has been involved in research projects related to estimation theory, noise reduction, optimal filtering, and pattern recognition. He developed SNR estimator for antenna diversity combining, singletrial subspace-based technique for EEG extraction form brain backgroun noise, and introduced a subspace-based data glove system for online signature verification. His present research interest is in brain signal processing, image enhancement, and pattern recognition. Currently, he is Associate Professor at the PETRONAS University of Technology. He is IEEE senior member. 